\title{
THE TRANSIT LIGHT CURVE PROJECT. II. TWO TRANSITS OF THE EXOPLANET OGLE-TR-111 ${ }^{1}$
}

\author{
Joshua N. Winn, ${ }^{2}$ Matthew J. Holman, ${ }^{3}$ and Cesar I. Fuentes ${ }^{3}$ \\ Received 2006 August 14; accepted 2006 September 19
}

\begin{abstract}
As part of our ongoing effort to measure exoplanet sizes and transit times with greater accuracy, we present $I$-band observations of two transits of OGLE-TR-111b. The photometry has an accuracy of $0.15 \%-0.20 \%$ and a cadence of $1-2$ minutes. We derive a planetary radius of $R_{P}=1.067 \pm 0.054 R_{\text {Jup }}$ and a stellar radius of $R_{S}=0.831 \pm 0.031 R_{\odot}$. The uncertainties are dominated by errors in the photometry rather than by systematic errors arising from uncertainties in the limb-darkening function or the stellar mass. Both the stellar radius and the planetary radius are in agreement with theoretical expectations. The transit times are accurate to within $30 \mathrm{~s}$ and allow us to refine the estimate of the mean orbital period: $4.0144479 \pm 0.0000041$ days.
\end{abstract}

Key words: planetary systems - stars: individual (OGLE-TR-111) — techniques: photometric

Online material: machine-readable table

\section{INTRODUCTION}

The aim of the Transit Light Curve (TLC) project is to gather accurate photometry during the transits of exoplanets across the disks of their parent stars. The immediate scientific harvest is the improved accuracy with which the basic system parameters are known. High-accuracy, high-cadence transit photometry allows for the determination of the stellar radius, the planetary radius, and the orbital inclination for an assumed value of the stellar mass (see, e.g., Seager \& Mallén-Ornelas [2003] for a discussion of the theory and Brown et al. [2001] for the most famous example of a transit light curve). These parameters are interesting in their own right, and are important in the interpretation of other measurements such as reflected light (Rowe et al. 2006), thermal emission (Charbonneau et al. 2005; Deming et al. 2005), atmospheric absorption (Charbonneau et al. 2002; Vidal-Madjar et al. 2003), and the Rossiter-McLaughlin effect (Queloz et al. 2000; Winn et al. 2005; Wolf et al. 2007).

In the longer term, repeated observations of transits will reduce the uncertainties in the system parameters (and especially the orbital period) still further. More interestingly, it may be possible to detect additional transiting objects, satellites, rings, or even reflected light by combining the data from many individual light curves. In addition, the existence of hitherto-undetected planets and satellites might be betrayed by periodic patterns in the measured times of midtransit or variations in the orbital inclination (Miralda-Escudé 2002; Holman \& Murray 2005; Agol et al. 2005).

The TLC project is currently in its initial phase, in which we are observing all of the known transiting exoplanets, refining the estimates of each system's parameters, and assessing the feasibility of continued long-term monitoring. We have previously reported on observations of the exoplanet XO-1b (Holman et al. 2006). In this paper we present TLC results for OGLE-TR-111b.

The periodic 2\% dimming events of the star OGLE-TR-111 were discovered by Udalski et al. (2002) in a survey for transiting planets within a rich star field in Carina. Spectroscopic follow-up by Pont et al. (2004) revealed a periodic Doppler shift, confirm-

\footnotetext{
${ }^{1}$ This paper includes data gathered with the $6.5 \mathrm{~m}$ Magellan Telescopes located at Las Campanas Observatory, Chile.

${ }^{2}$ Department of Physics, and Kavli Institute for Astrophysics and Space Research, Massachusetts Institute of Technology, Cambridge, MA, USA.

${ }^{3}$ Harvard-Smithsonian Center for Astrophysics, Cambridge, MA, USA.
}

ing that the dimming events were caused by the transits of a Jovian planet. Santos et al. (2006) obtained optical spectra with a higher signal-to-noise ratio to study the properties of the host star, which is an early $\mathrm{K}$ dwarf with roughly solar metallicity. The orbital period is just over 4 days, which is the longest period among the five exoplanets identified in the Optical Gravitational Lensing Experiment (OGLE) survey but is typical of the periods of the "hot Jupiters" that have been discovered in abundance in radial velocity surveys. For this reason, Pont et al. (2004) referred to OGLE-TR-111 as the "missing link" between the OGLE survey and the radial velocity surveys. Initially, it was thought that the two surveys were yielding discrepant results because of the shorter periods of the OGLE objects, but this is now understood as a selection effect (Pont et al. 2005; Gaudi et al. 2005; Gould et al. 2006).

This paper is organized as follows. We describe the observations in $\S 2$ and the photometric procedure in $\S 3$. In $\S 4$ we describe the techniques we used to estimate the physical and orbital parameters. In $\S 5$ we give the results, and $\S 6$ is a brief summary.

\section{OBSERVATIONS}

We observed two transits of OGLE-TR-111 (on UT 2006 February 21 and March 5) corresponding to epochs $E=363$ and 366 of an updated ephemeris based on OGLE data that were provided by A. Udalski (2005, private communication):

$$
T_{c}(E)=2,452,330.46228[\mathrm{HJD}]+E(4.014442 \text { days }) .
$$

We used the Inamori Magellan Areal Camera and Spectrograph (IMACS) on the $6.5 \mathrm{~m}$ Baade (Magellan I) telescope at Las Campanas Observatory, in Chile. IMACS has two cameras differing in focal length. We used the longer $\mathrm{f} / 4.3$ camera because we preferred the smaller pixel scale, and the field of view was still large enough to encompass multitudes of comparison stars. Photometry is improved with small pixels not only because of the better spatial sampling of the point-spread function (PSF), but also because spreading the starlight over many pixels averages down the pixel-to-pixel sensitivity variations and increases the maximum exposure time due to saturation. The IMACS detector is a mosaic of eight $2 \mathrm{~K} \times 4 \mathrm{~K}$ SITe back-illuminated and thinned CCDs with $15 \mu \mathrm{m}$ pixels, giving a pixel scale of $0.111^{\prime \prime}$ and a field of view of $15.4^{\prime}$. To reduce the readout time, we read only onethird of each chip, corresponding to the central $15.4^{\prime} \times 5.1^{\prime}$ of the 
mosaic. The readout time was approximately $45 \mathrm{~s}$, and the readout noise was about $5 e^{-}$. We observed through the Cerro Tololo InterAmerican Observatory $I$-band filter, the reddest broadband filter in routine use on IMACS, in order to minimize the effect of colordependent atmospheric extinction on the relative photometry and to minimize the effect of limb darkening on the transit light curve. On each of the two nights, we observed OGLE-TR-111 for approximately $6 \mathrm{hr}$, bracketing the predicted midpoint of the transit. We also obtained dome-flat exposures and zero-second (bias) exposures at the beginning of each night.

On the night of UT 2006 February 21 we observed under clear skies through an air mass ranging from 1.2 to 1.6. The seeing was generally good but variable, from $0.5^{\prime \prime}$ to $1.0^{\prime \prime}$. We used an exposure time of $60 \mathrm{~s}$. Although the sky conditions were excellent, three factors degraded the photometry to some degree. First, although we attempted to keep the image registration constant throughout the night (thereby consistently detecting the light from each star on the same set of pixels), this was not possible due to occasional failures of the guide probe control software. The changes in registration had a noticeable effect on the relative photometry, as described further in $\S 3$. Second, the diffraction spike from a nearby bright star swept through the position of OGLE-TR-111 on two occasions, with noticeable effects on the photometry. Third, the atmospheric dispersion corrector (ADC) was not functioning correctly, causing color-dependent effects in the stellar images.

On the night of UT 2006 March 5 the skies were also clear, and the seeing was more consistent at approximately $0.9^{\prime \prime}$ all night. We used a shorter exposure time of $30 \mathrm{~s}$. As in February, there were occasional crashes of the guiding software, and a diffraction spike swept through the position of OGLE-TR-111. However, on this night the ADC was functioning properly.

\section{DATA REDUCTION}

We used standard IRAF ${ }^{4}$ procedures for the overscan correction, trimming, bias subtraction, and flat-field division. Because the images were too crowded for aperture photometry, we used the method of image subtraction as implemented by Alard \& Lupton (1998) and Alard (2000). Specifically, we used version 2.2 of the ISIS image subtraction package that was written and kindly made public by $\mathrm{C}$. Alard. In this method, all of the images from a given night are registered to a common pixel frame, and a reference image is created by combining a subset $(\approx 10 \%)$ of the images with the best seeing. For each individual image, a convolution kernel is determined that brings the image into best agreement with the reference image. Then the difference is computed between the appropriately convolved image and the reference image. The advantage of this method is that photometry is simplified on the difference images, because most stars are not variable stars and thus the complex and crowded background is eliminated. It is still necessary to compute the flux of the variable stars on the reference image (taking into account any neighboring stars), but this need only be done once, and the task is facilitated by the good spatial resolution and high signal-to-noise ratio of the reference image. Thus, the measurement of the relative flux $f(t)$ takes the form

$$
f(t)=1+\frac{\Delta f(t)}{f_{\text {ref }}},
$$

where $f_{\text {ref }}$ is measured on the reference image and $\Delta f(t)$ is measured on the difference images.

\footnotetext{
4 The Image Reduction and Analysis Facility (IRAF) is distributed by the National Optical Astronomy Observatory, which is operated by the Association of Universities for Research in Astronomy, Inc., under cooperative agreement with the National Science Foundation.
}

We performed photometry of OGLE-TR-111 along with 18 other stars of comparable brightness for quality control. We tried two different methods for performing the photometry on the difference images: IRAF-based aperture photometry and ISIS-based profile-fitting photometry. For the March data, superior results (in the sense of a smaller standard deviation in the light curves of the comparison stars) were obtained with profile-fitting photometry, whereas for the February data, superior results were obtained with aperture photometry. We suspect that the reason for the difference is that the February data were taken under more variable seeing and without proper atmospheric dispersion correction. Because of these differing conditions, we adopted the aperture results for February and the profile-fitting results for March, rather than requiring the same procedure to be used on all of the data. To determine $f_{\text {ref }}$ for each star, we performed profile-fitting photometry on the reference image for each night.

The uncertainty in each data point arises from two sources: the uncertainty in the difference flux $\Delta f$ and the uncertainty in the reference flux $f_{\text {ref }}$. We estimated the uncertainty in the difference flux based on Poisson statistics. We estimated the uncertainty in the reference flux based not only on Poisson statistics but also by the spread in the values obtained when using different choices for the stars used to determine the PSF and other parameters relating to the profile photometry. This latter source of systematic error was $1.5 \%$ for the February data and $1 \%$ for the March data, which dominated the Poisson error in both cases. However, adjustments in $f_{\text {ref }}$ affect all of the points from a given night in the same way; the net effect is a small modification of the transit depth. For example, for OGLE-TR-111 the transit depth is approximately $2 \%$. The effect of increasing $f_{\text {ref }}$ by $1 \%$ is to decrease the transit depth by $(0.02 \times 0.01)$ or $2 \times 10^{-4}$. We discuss this systematic error further in $\oint 5$.

Abrupt jumps in the photometry by $\sim 0.5 \%$ were evident when the diffraction spike from a nearby star intruded on the position of OGLE-TR-111 and when the telescope pointing changed (presumably due to flat-fielding errors). We discarded the data that were affected by the diffraction spike. For the February data the pointing changed approximately 15 minutes prior to ingress, and again just after egress; in our subsequent analysis we used only the data obtained between those times. Most of the March data were taken with a common pointing, except for an interval of 30 minutes after egress, which occurred after a guider failure and before the pointing could be restored to its former value. Those 30 minutes of data were not considered further.

Although the image subtraction method removes the first-order effects of extinction by scaling all of the images to a common flux level before subtraction, residual color-dependent effects are not removed. Stars of different colors are extinguished by different amounts through a given air mass. For this reason we applied a residual extinction correction to the data. The correction function was determined as part of the model-fitting procedure and is described in $\S 4$. The final photometry is given in Table 1 and is plotted in Figure 1. In Figure 1 (bottom) our composite light curve is compared to that of the OGLE survey data. The uncertainties given in Table 1 are the uncertainties in the difference fluxes after multiplying by a factor specific to each night such that $\chi^{2} / N_{\text {dof }}=1$ for the best-fitting model. (Our intention was not to test the model but rather to determine the appropriate relative weights for the data points.) The scaling factors were 1.32 for the February data and 1.01 for the March data.

\section{THE MODEL}

Our model for the system is based on a star (with mass $M_{S}$ and radius $R_{S}$ ) and a planet (with mass $M_{P}$ and radius $R_{P}$ ) in a circular 
TABLE 1

Рнотомetry of OGLE-TR-111

\begin{tabular}{|c|c|c|}
\hline HJD & Relative Flux & Uncertainty \\
\hline $2,453,787.63734 \ldots$ & 0.99982 & 0.00158 \\
\hline $2,453,787.63853 \ldots \ldots \ldots \ldots \ldots \ldots \ldots \ldots$ & 1.00064 & 0.00152 \\
\hline $2,453,787.63982 \ldots \ldots \ldots \ldots \ldots \ldots \ldots \ldots$ & 0.99996 & 0.00151 \\
\hline $2,453,787.64097 \ldots \ldots \ldots \ldots \ldots \ldots \ldots \ldots$ & 0.99932 & 0.00156 \\
\hline 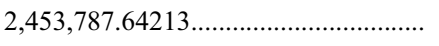 & 0.99863 & 0.00154 \\
\hline
\end{tabular}

Notes.-The time stamps represent the Heliocentric Julian Date at the time of midexposure. The uncertainty estimates are based on the procedures described in $\S 2$. This table appears in entirety in the electronic version of the Astronomical Journal. A portion is shown here to illustrate its format. The data are also available from the authors on request. orbit $^{5}$ with period $P$ and inclination $i$ relative to the sky plane. We define the coordinate system such that $0^{\circ} \leq i \leq 90^{\circ}$. We allow each transit to have an independent value of $T_{c}$, the transit midpoint, rather than forcing them to be separated by an integral number of orbital periods. This is because we seek to measure or bound any timing anomalies that may indicate the presence of moons or additional planets in the system. Thus, the period $P$ is relevant to the model only through the connection between the total mass and the orbital semimajor axis. We fix $P=4.01444$ days,

\footnotetext{
${ }^{5}$ A circular orbit is a reasonable simplifying assumption because it is expected that there has been sufficient time for tides to have damped out any initial eccentricity, in the absence of a third body (see, e.g., Rasio et al. 1996; Trilling 2000; Dobbs-Dixon et al. 2004).
}
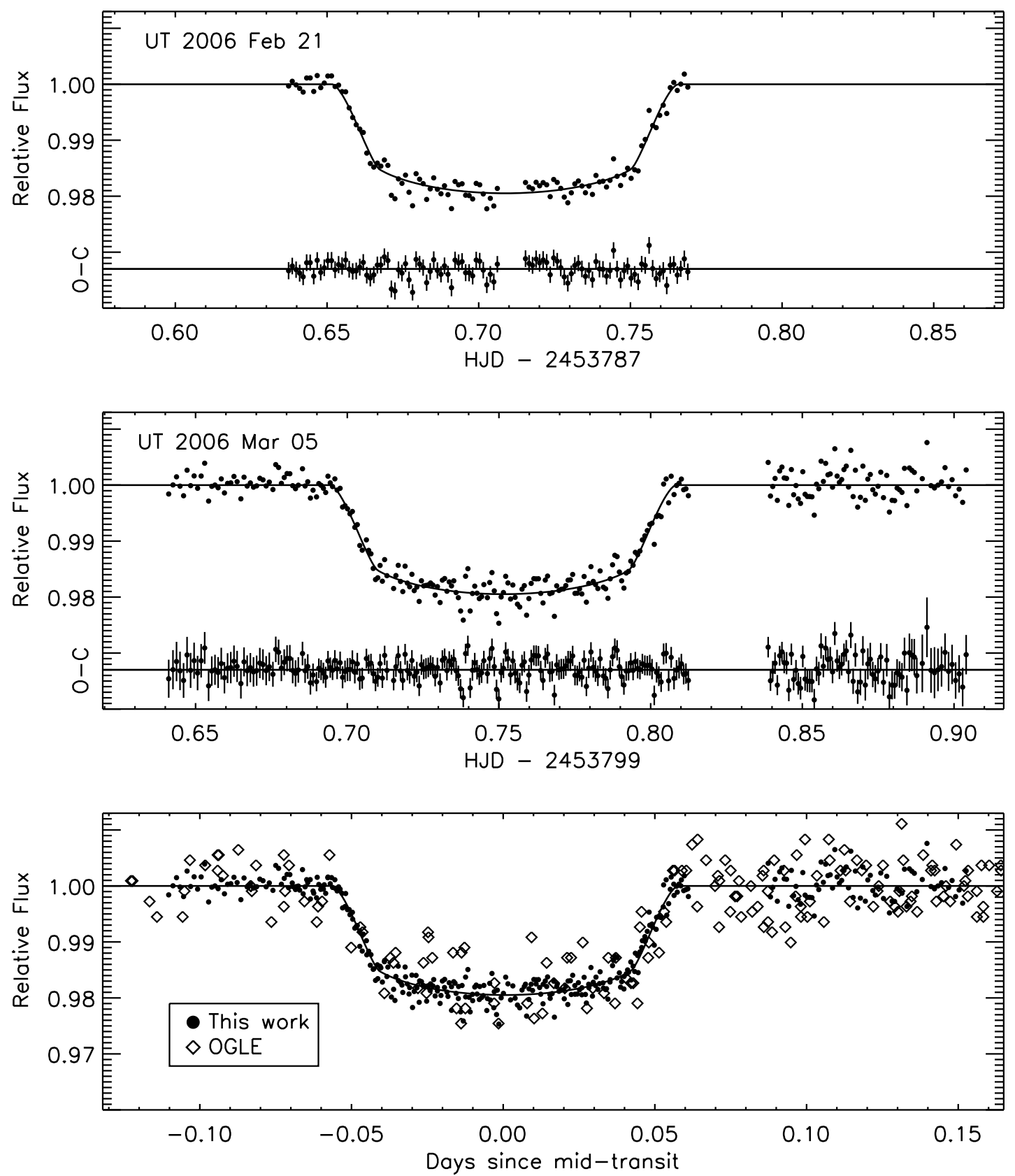

FIG. 1.-Relative $I$-band photometry of OGLE-TR-111. The best-fitting model is shown as a solid line. The residuals (observed - calculated) and the rescaled $1 \sigma$ error bars are also shown. The residuals have zero mean but are offset by a constant flux to appear beneath each light curve, for clarity. The rms residuals are $0.15 \%$ and $0.2 \%$ for the February and the March data, respectively. The bottom panel shows the composite light curve and also a composite light curve based on the OGLE survey data. 
the value kindly provided by A. Udalski (2005, private communication) based on several seasons of OGLE data.

The stellar mass cannot be determined from transit photometry alone. Furthermore, the values of $R_{S}$ and $R_{P}$ that are inferred from the photometry are covariant with the stellar mass; for a fixed period $P$ the photometric transit depends almost exactly on the combinations $R_{S} / M_{S}^{1 / 3}$ and $R_{P} / M_{S}^{1 / 3}$. Our approach was to fix $M_{S}=0.81 M_{\odot}$, the value reported by Santos et al. (2006) based on an analysis of the stellar spectrum (i.e., the observed effective temperature, surface gravity, and metallicity were compared to theoretical H-R diagrams). We then used the scaling relations $R_{P} \propto M_{S}^{1 / 3}$ and $R_{S} \propto M_{S}^{1 / 3}$ to estimate the systematic error due to the uncertainty in $M_{S}$. The planetary mass $M_{P}$ is nearly irrelevant to the model (except for its minuscule effect on the relation between the orbital period and the semimajor axis), but for completeness we use the value $M_{P}=0.52 M_{\text {Jup }}$ reported by Santos et al. (2006).

To calculate the relative flux as a function of the projected separation of the planet and the star, we assumed the limb-darkening law to be linear:

$$
\frac{I(\mu)}{I(1)}=1-u(1-\mu)
$$

where $I$ is the intensity and $\mu$ is the cosine of the angle between the line of sight and the normal to the stellar surface. Adding a parameter to the limb-darkening law by making it a quadratic function of $(1-\mu)$ did not significantly improve the fit, and hence is not well justified by the data alone. We employed the analytic formulae of Mandel \& Agol (2002) to compute the integral of the intensity over the exposed portion of the stellar disk. The limb-darkening parameter $u$ was a variable in the model, but we applied an a priori constraint to require a reasonable level of agreement with theoretical expectations for limb darkening, as described below. Each transit is also described with two additional parameters: the out-of-transit flux $f_{\text {oot }}$ and a residual extinction coefficient $k$. The latter is defined such that the observed flux is proportional to $\exp (-k z)$.

In total, there are 10 adjustable parameters describing 386 photometric data points. The parameters are $R_{S}, R_{P}$, and $i$; the two values of $T_{c}$; the limb-darkening parameter $u$; and the values of $f_{\text {oot }}$ and $k$ for each transit. Our goodness-of-fit parameter is

$$
\chi^{2}=\sum_{j=1}^{386}\left[\frac{f_{j}(\mathrm{obs})-f_{j}(\mathrm{calc})}{\sigma_{j}}\right]^{2}+\left(\frac{u-u_{\mathrm{th}}}{\sigma_{u}}\right)^{2},
$$

where $f_{j}$ (obs) is the flux observed at time $j, \sigma_{j}$ is the corresponding uncertainty, and $f_{j}$ (calc) is the calculated value. The last term is the a priori constraint on the limb-darkening parameter. The theoretical value $u_{\text {th }}=0.597$ comes from fits by Claret (2000) to an ATLAS plane-parallel stellar atmosphere model of R. Kurucz, for a star with $T_{\text {eff }}=5000 \mathrm{~K}, \log g=4.5(\mathrm{cgs})$, and $[\mathrm{Fe} / \mathrm{H}]=0.2$. We set $\sigma_{u}=0.081$, corresponding to the requirement that the limbto-center intensity ratio $(1-u)$ should be within about $20 \%$ of the calculated value. (We also investigated the effects of tightening, modifying, and dropping this a priori constraint, as discussed in $\S 5$.) As noted in $\S 3$, we took the uncertainties $\sigma_{j}$ to be the calculated uncertainties after multiplication by a factor specific to each night, such that $\chi^{2} / N_{\text {dof }}=1$ when each night's data were fitted individually.

We began by finding the values of the parameters that minimize $\chi^{2}$, using the venerable AMOEBA algorithm (Press et al. 1992 , p. 408). Then we estimated the a posteriori joint proba-
TABLE 2

\begin{tabular}{|c|c|c|}
\hline $\begin{array}{l}\text { Parameter } \\
\text { (1) }\end{array}$ & $\begin{array}{l}\text { Value } \\
\text { (2) }\end{array}$ & $\begin{array}{c}\text { Uncertainty } \\
\text { (3) }\end{array}$ \\
\hline$R_{S}\left(R_{\odot}\right)$ & 0.831 & 0.031 \\
\hline$R_{P} / R_{\text {Jup }} \cdots \cdots \cdots$ & 1.067 & 0.054 \\
\hline$R_{P} / R_{S}$ & 0.132 & 0.002 \\
\hline$i(\operatorname{deg})$ & 88.1 & 0.5 \\
\hline$b$ & 0.39 & 0.09 \\
\hline$t_{\mathrm{IV}}-t_{\mathrm{I}}(\mathrm{hr}) \ldots \ldots$ & 2.743 & 0.033 \\
\hline$t_{\mathrm{II}}-t_{\mathrm{I}}$ (minutes) & 22.2 & 2.0 \\
\hline$T_{c}(363)(\mathrm{HJD})$ & $2,453,787.70854$ & 0.00035 \\
\hline$T_{c}(366)(\mathrm{HJD}) \ldots \ldots$ & $2,453,799.75138$ & 0.00032 \\
\hline и & 0.49 & 0.05 \\
\hline
\end{tabular}

System Parameters of OGLE-TR-111

Notes.-The parameter values in col. (2) are the median values $p_{\text {med }}$ of the MCMC distributions. The quoted uncertainty is the average of $\left|p_{\text {med }}-p_{\mathrm{lo}}\right|$ and $\left|p_{\text {med }}-p_{\text {hi }}\right|$, where $p_{\text {lo }}$ and $p_{\text {hi }}$ are the lower and upper $68 \%$ confidence limits. (The cumulative probability for values below $p_{\mathrm{lo}}$ is $16 \%$, and the cumulative probability for values above $p_{\text {hi }}$ is also $16 \%$.) For the stellar mass, we use the value $M_{S}=0.81$ $\pm 0.02 M_{\odot}$ from Santos et al. (2006). The systematic errors due to the uncertainties in the stellar mass, the limb-darkening function, and the reference flux are considerably smaller than the statistical errors and are not included here (see the text).

bility distribution for the parameter values using a Markov Chain Monte Carlo (MCMC) technique (for a brief introduction, consult Appendix A of Tegmark et al. 2004). In this method a chain of points in parameter space is generated from an initial point by iterating a jump function, which in our case was the addition of a Gaussian random number to each parameter value. If the new point has a lower $\chi^{2}$ than the previous point, the jump is executed; if not, the jump is only executed with probability exp $\left(-\Delta \chi^{2} / 2\right)$. We set the typical sizes of the random perturbations such that $\sim 20 \%$ of jumps are executed. We created 10 independent chains, each with 500,000 points, starting from random initial positions. The first 100,000 points were not used to minimize the effect of the initial condition. The correlation lengths were $\sim 10^{3}$ steps for the highly covariant parameters $R_{P}, R_{S}$, and $b$, and a few hundred steps for the other parameters. The Gelman \& Rubin (1992) $R$-statistic was within $0.1 \%$ of unity for each parameter, a sign of good mixing and convergence.

\section{RESULTS}

The model that minimizes $\chi^{2}$ is plotted as a solid line in Figure 1 . The optimized residual extinction correction has been applied to the data that are plotted in Figure 1, and to the data that are given in Table 1. The differences between the observed fluxes and the model fluxes are also shown beneath each light curve.

Table 2 gives the estimated values and uncertainties for each parameter based on the MCMC analysis. It also includes some useful derived quantities: the impact parameter $b=a \cos i / R_{S}$ (where $a$ is the semimajor axis); the time between first and last contact $\left(t_{\mathrm{IV}}-t_{\mathrm{I}}\right)$; and the time between first and second contact $\left(t_{\mathrm{II}}-t_{\mathrm{I}}\right)$. Figure 2 shows the estimated a posteriori probability distributions for the especially interesting parameters $R_{S}, R_{P}$, and $b$, along with some of the two-dimensional correlations involving those parameters. Although the distributions shown in Figure 2 are somewhat asymmetric about the median, Table 2 reports only the median $p_{\text {med }}$ and a single number $\sigma_{p}$ characterizing the width of the distribution. The value of $\sigma_{p}$ is the average of $\left|p_{\text {med }}-p_{\text {hi }}\right|$ and $\left|p_{\text {med }}-p_{\text {lo }}\right|$, where $p_{\text {lo }}$ and $p_{\text {hi }}$ are the lower and upper $68 \%$ confidence limits. We refer to $\sigma_{p}$ as the "statistical error" to distinguish it from the sources of systematic error discussed below. 

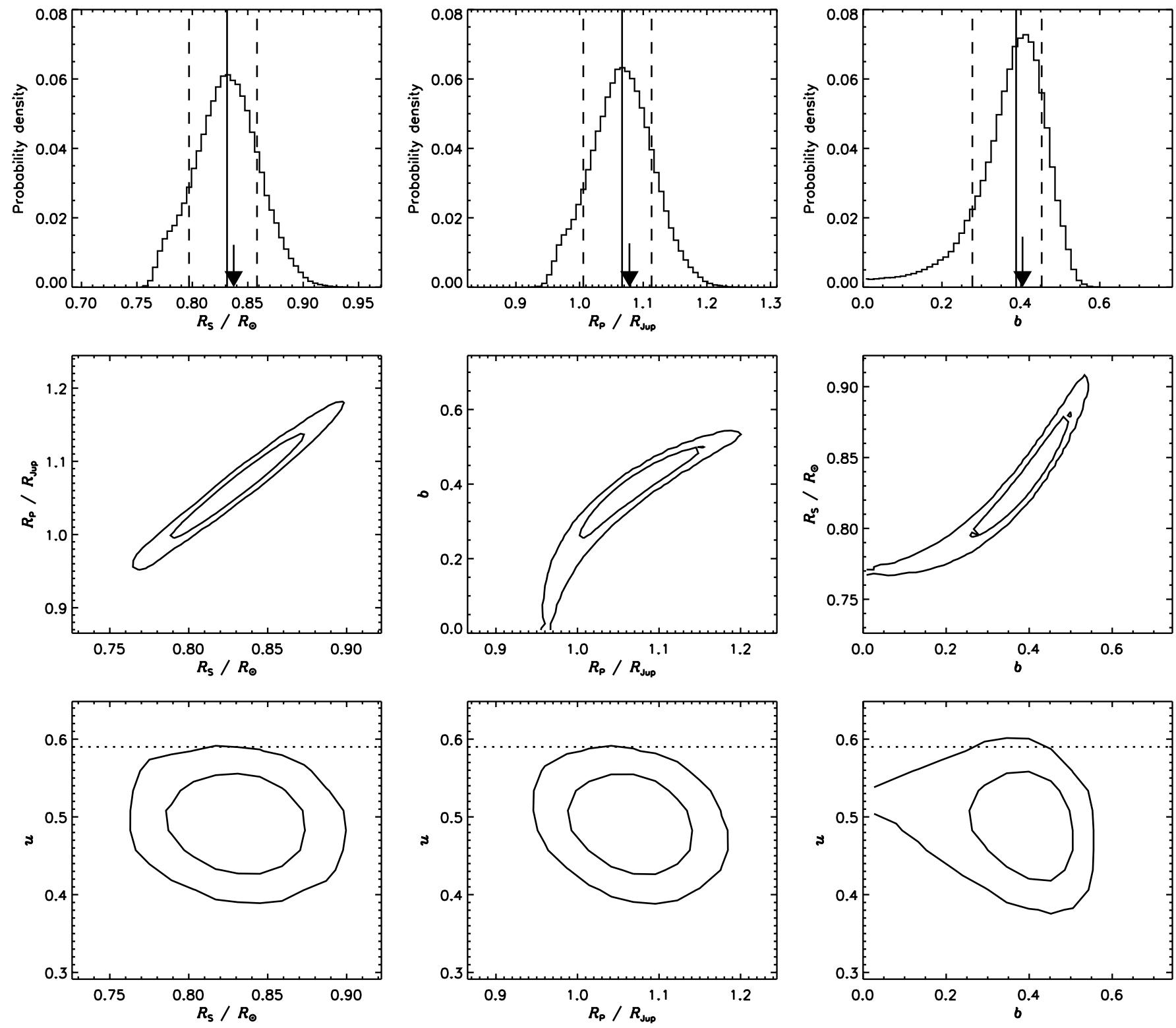

FIG. 2.-Top panels: Probability distributions for the stellar radius $R_{S}$, planetary radius $R_{P}$, and impact parameter $b \equiv a \cos i / R_{S}$ based on the MCMC simulations. The arrows mark the values of the parameters that minimize $\chi^{2}$. A solid line marks the median of each distribution, and the dashed lines mark the $68 \%$ confidence limits. Middle and bottom panels: Joint probability distributions of those parameters with the strongest correlations. The contours are isoprobability contours enclosing $68 \%$ and $95 \%$ of the points in the Markov chains. The dotted lines indicate the value of the limb-darkening parameter calculated by Claret (2000) based on an ATLAS model of a star with $T_{\text {eff }}=5000 \mathrm{~K}, \log g=4.5(\mathrm{cgs}),[\mathrm{Fe} / \mathrm{H}]=0.2$, and $\xi_{t}=2 \mathrm{~km} \mathrm{~s}^{-1}$.

There are several sources of systematic error that are not taken into account by the MCMC analysis. The first is the systematic error that was already discussed in $\S 4$ : the covariance between the stellar mass $M_{S}$ and both of the parameters $R_{P}$ and $R_{S}$. For a fixed value of $P$, the photometric signal depends on the combinations $R_{P} / M_{S}^{1 / 3}$ and $R_{S} / M_{S}^{1 / 3}$. A value for $M_{S}$ must be chosen on other grounds. We adopted the value $M_{S}=0.81 M_{\odot}$ based on the most recent analysis of the spectrum of OGLE-TR-111 by Santos et al. (2006). Based on their measurements of the equivalent widths of 38 iron absorption lines and the wings of the $\mathrm{H} \alpha$ absorption profile, those investigators concluded that the uncertainty in $M_{S}$ was only $2.5 \%$. The corresponding fractional error in $R_{P}$ and $R_{S}$ due to the covariance is only $0.8 \%$, which is negligible when compared to the statistical errors of $4 \%$ and $5 \%$, respectively. Another way to state this result is that the uncertainty in the stellar mass would need to be $\gtrsim 10 \%$ (i.e., 4 times larger than the uncertainty quoted by Santos et al. 2006) for the resulting systematic error to be comparable to the statistical error.

A second source of systematic error is the bias due to an incorrect choice of limb-darkening function. Neither the appropriate functional form of the limb-darkening function nor the value of the limb-darkening coefficient is known with certainty. One can calculate the limb-darkening function based on stellar atmosphere models, but the uncertainties in both the stellar parameters and the atmosphere models make it difficult to quantify the uncertainty in the results. We attempted to account for this uncertainty with the a priori constraint on $u$ that was described in $\S 4$; here we describe the results of some tests of the sensitivity of our results on the treatment of limb darkening. For brevity, we describe only the variation in $R_{P}$ under different assumptions, because we have found that this parameter shows the greatest sensitivity to changes in the assumed limb-darkening law. If $u$ 
is held fixed at the Claret (2000) value of 0.597 , then $R_{P}$ is decreased by $1.3 \%$ relative to the value given in Table 1 . If $u$ is allowed to vary with no constraint, $R_{P}$ rises by $0.8 \%$. Using a quadratic limb-darkening law instead of a linear law, $R_{P}$ decreases by $2.5 \%$ if the coefficients are fixed at the Claret (2000) values, and by $0.5 \%$ if they vary freely. The four-parameter "nonlinear" law gives essentially the same results as the quadratic law, and the PHOENIX-based coefficients give essentially the same results as the ATLAS coefficients. We conclude that the systematic error in $R_{P}$ due to the choice of limb-darkening law is a few percent at most, which is smaller than the statistical error.

A third source of systematic error is in the measurement of the flux of OGLE-TR-111 on the reference image of the imagesubtraction photometric procedure (see $\S 3$ ). For example, if the reference flux $f_{\text {ref }}$ is erroneously large, then the transit depth (and the inferred value of $R_{P} / R_{S}$ ) will be erroneously small. We assessed the size of this effect by refitting the data after adjusting the value of $f_{\text {ref }}$ for the March data (which dominates $\chi^{2}$ ) upward or downward by the estimated error. The results for $R_{P}$ and $R_{S}$ are altered by $\lesssim 0.5 \%$, a change that can be neglected in light of the larger errors determined previously.

We believe that these three effects are the largest sources of systematic error, and we have shown that all of them are smaller than the statistical errors in the photometry. Therefore, unless the uncertainty in $M_{S}$ has been grossly underestimated by Santos et al. (2006), we conclude that there is considerable scope for improvement in the system parameters through additional photometry.

Our value of $R_{P}=(1.067 \pm 0.054) R_{\text {Jup }}$ for the planetary radius is in agreement with previous estimates. Pont et al. (2004) found $R_{P}=1.00_{-0.06}^{+0.13} R_{\text {Jup }}$ based on the OGLE photometry, and Santos et al. (2006) refined this value to $R_{P}=(0.97 \pm 0.06) R_{\text {Jup }}$. These values, in turn, have already shown to be in broad agreement with theoretical expectations for "hot Jupiters" (see, e.g., Baraffe et al. 2005). It might seem surprising that our estimate is hardly more precise than that of Santos et al. (2006), despite our superior photometry. However, the comparison is misleading. The time sampling and signal-to-noise ratio of the OGLE photometry were insufficient for reliable measurements of the ingress and egress durations. Consequently, it was necessary for previous transit light-curve fitters to adopt an a priori value of $R_{S}$ in addi- tion to $M_{S}$. We have been able to derive $R_{S}$ from the photometry, subject only to the weak covariance with the assumed value of $M_{S}$. The agreement between our result $R_{S}=0.831 \pm 0.031 R_{\odot}$ and the spectroscopic estimate of $0.83 \pm 0.02 R_{\odot}$ is therefore a new and important cross-check on the system parameters.

The uncertainties in the transit times are about $30 \mathrm{~s}$, and these uncertainties are not correlated with those of any of the other model parameters except for the residual air mass correction. The interval between the two transits was 12.0428(5) days, where the number in parentheses is the $1 \sigma$ error in the last digit. This corresponds to an "instantaneous period" of 4.0143(2) days. We refined the precision of the transit ephemeris through a simultaneous fit to all of the OGLE photometry and our own photometry, assuming the period to be uniform. Only the orbital period $P$ and one particular time of transit $T_{c}$ were allowed to vary; all of the rest of the parameters were held fixed at the values given in Table 1 . The refined ephemeris is

$$
\begin{aligned}
T_{c} & =2,453,799.7516 \pm 0.0002[\mathrm{HJD}] \\
P & =4.0144479 \pm 0.0000041 \text { days. }
\end{aligned}
$$

\section{SUMMARY}

Through observations of two closely spaced transits of the exoplanet OGLE-TR-111b, we have improved on the estimates of the system parameters. The improvement comes not only from an overall increase in the signal-to-noise ratio but also from the ability to resolve the ingress and egress and thereby determine the stellar radius photometrically. Our results confirm the previous estimates of the stellar and planetary radii and are subject to a smaller systematic error. We have also provided a more precise transit ephemeris. All of these results will help with future observations and interpretations of this system.

We have benefitted from helpful consultations with D. Sasselov on limb darkening, G. Torres on stellar mass determination, and S. Gaudi on parameter estimation. We thank A. Udalski for providing an updated OGLE ephemeris and A. Roussanova for proofreading the manuscript.

\section{REFERENCES}

Agol, E., Steffen, J., Sari, R., \& Clarkson, W. 2005, MNRAS, 359, 567

Alard, C. 2000, A\&AS, 144, 363

Alard, C., \& Lupton, R. H. 1998, ApJ, 503, 325

Baraffe, I., Chabrier, G., Barman, T. S., Selsis, F., Allard, F., \& Hauschildt, P. H. 2005, A\&A, 436, L47

Brown, T. M., Charbonneau, D., Gilliland, R. L., Noyes, R. W., \& Burrows, A. 2001, ApJ, 552, 699

Charbonneau, D., Brown, T. M., Noyes, R. W., \& Gilliland, R. L. 2002, ApJ, 568,377

Charbonneau, D., et al. 2005, ApJ, 626, 523

Claret, A. 2000, A\&A, 363, 1081

Deming, D., Seager, S., Richardson, L. J., \& Harrington, J. 2005, Nature, 434, 740

Dobbs-Dixon, I., Lin, D. N. C., \& Mardling, R. A. 2004, ApJ, 610, 464

Gaudi, B. S., Seager, S., \& Mallén-Ornelas, G. 2005, ApJ, 623, 472

Gelman, A., \& Rubin, D. B. 1992, Stat. Sci., 7, 457

Gould, A., Dorsher, S., Gaudi, B. S., \& Udalski, A. 2006, Acta Astron., 56, 1

Holman, M. J., \& Murray, N. W. 2005, Science, 307, 1288

Holman, M. J., et al. 2006, ApJ, 652, 1715

Mandel, K., \& Agol, E. 2002, ApJ, 580, L171

Miralda-Escudé, J. 2002, ApJ, 564, 1019
Pont, F., Bouchy, F., Melo, C., Santos, N. C., Mayor, M., Queloz, D., \& Udry, S. 2005, A\&A, 438, 1123

Pont, F., Bouchy, F., Queloz, D., Santos, N. C., Melo, C., Mayor, M., \& Udry, S. 2004, A\&A, 426, L15

Press, W. H., Teukolsky, S. A., Vetterling, W. T., \& Flannery, B. P. 1992, Numerical Recipes in C (Cambridge: Cambridge Univ. Press)

Queloz, D., Eggenberger, A., Mayor, M., Perrier, C., Beuzit, J. L., Naef, D., Sivan, J. P., \& Udry, S. 2000, A\&A, 359, L13

Rasio, F. A., Tout, C. A., Lubow, S. H., \& Livio, M. 1996, ApJ, 470, 1187

Rowe, J. F., et al. 2006, ApJ, 646, 1241

Santos, N. C., et al. 2006, A\&A, 450, 825

Seager, S., \& Mallén-Ornelas, G. 2003, ApJ, 585, 1038

Tegmark, M., et al. 2004, Phys. Rev. D, 69, 103501

Trilling, D. E. 2000, ApJ, 537, L61

Udalski, A., Szewczyk, O., Żebrun, K., Pietrzyński, G., Szymański, M., Kubiak, M., Soszyński, I., \& Wyrzykowski, Ł. 2002, Acta Astron., 52, 317

Vidal-Madjar, A., Lecavelier des Etangs, A., Désert, J.-M., Ballester, G. E., Ferlet, R., Hébrard, G., \& Mayor, M. 2003, Nature, 422, 143

Winn, J. N., et al. 2005, ApJ, 631, 1215

Wolf, A., et al. 2007, ApJ, in press 